\title{
A pilot study employing hepatic intra-arterial irinotecan injection of drug-eluting beads as salvage therapy in liver metastatic colorectal cancer patients without extrahepatic involvement: the first southern Italy experience
}

This article was published in the following Dove Press journal:

OncoTargets and Therapy

I 2 December 2016

Number of times this article has been viewed

Girolamo Ranieri, ' Artor

Niccoli Asabella, ${ }^{2}$ Corinna

Altini, ${ }^{2}$ Vito Fazio, ${ }^{1}$

Luciana Caporusso,'

Ilaria Marech,' Gianluca

Vinciarelli,' Francesco

Macina,' Dario de Ceglia,'

Margherita Fanelli, ${ }^{2}$ Michele

Ammendola, ${ }^{3,4}$ Giuseppe

Rubini, ${ }^{2}$ Cosmo Damiano

Gadaleta'

'Diagnostic and Interventional Radiology Unit with Integrated

Section of Translational Medical Oncology, National Cancer Research

Centre, Istituto Tumori "Giovanni

Paolo II", ${ }^{2}$ Nuclear Medicine Unit,

University of Bari "Aldo Moro", Bari,

${ }^{3}$ Department of Medical and Surgery

Science, Clinical Surgery Unit, “Magna

Graecia" Medical School, Catanzaro,

${ }^{4}$ Surgery Unit, National Cancer

Research Centre, Istituto Tumori

"Giovanni Paolo II", Bari, Italy

Correspondence: Girolamo Ranieri Diagnostic and Interventional Radiology Unit with Integrated Section of

Translational Medical Oncology, National Cancer Research Centre, Istituto Tumori “Giovanni Paolo II", Viale Orazio

Flacco 65, 70I 24 Bari, Italy

Tel +393248050022

Email giroran@tiscali.it
Background: The main aim of this prospective study was to evaluate the efficacy of drug-eluting beads with irinotecan (DEBIRI) for liver metastases from colorectal cancer. Secondary aims were to evaluate survival and toxicity.

Methods: Twenty-five patients with metastases in $<50 \%$ of the liver and without extrahepatic involvement were enrolled. Treatment response assessment was performed by multidetector contrast enhancement computed tomography (MDCT) with evaluation of the enhancement pattern of the target lesion and tumor response rates according to modified Response Evaluation Criteria in Solid Tumors (mRECIST, Version 1.1). All adverse events were recorded by the Cancer Therapy Evaluation Program Common Terminology Criteria for Adverse Events, Version 3.0. Associations of tumor response and variables were calculated using the chi-squared test. Overall survival (OS) was calculated using the Kaplan-Meier method. Comparisons were made using the log-rank test.

Results: According to mRECIST, complete response (CR) was observed in $21.8 \%$ of patients, partial response (PR) in 13\%, stable disease (SD) in 52.2\% and progressive disease (PD) in $13 \%$ of patients. Response rate $(\mathrm{RR}=\mathrm{CR}+\mathrm{PR})$ was $34.8 \%$. No associations between treatment response and variables such as Dukes' classification, grading and Kras status were found $(P>0.05)$. The median OS was 37 months (95\% CI: 13.881 to 60.119). Cox regression model showed that neither site, Dukes' classification, grading, Kras status nor number of chemotherapy treatments pre-DEBIRI influenced the OS. The log-rank test showed no statistically significant difference in OS among patients who underwent 1,2 or 3 DEBIRI treatments $\left(\chi^{2}=2.831, P=0.09\right)$. In our study, the main toxicities included postembolization syndrome (PES), hypertransaminasemia and fever.

Conclusion: The favorable tumor response and the favorable toxicity profile make DEBIRI treatment a potential third-line therapy. Although further larger studies are needed to confirm these data, we can state that DEBIRI is an attractive emerging treatment in these patients.

Keywords: colorectal cancer, liver metastases, transarterial chemoembolization, DEBIRI

\section{Background}

CRC is the third most common cancer worldwide. ${ }^{1} \mathrm{Up}$ to $70 \%$ of its metastases occur in the liver, and LM represent a predominant cause $(50 \%)$ of death in these patients. ${ }^{2}$ Without any treatment, the median OS after detection of LM is $\sim 9$ months, depending 
on the extent of the disease at the time of diagnosis. ${ }^{3,4}$ In $\sim 30 \%$ of patients who develop LM, the metastatic disease will remain confined to the liver. ${ }^{4}$ Surgical resection is the standard treatment with a 5-year survival of only $25 \%-35 \%$ and recurrence appearance with a rate as high as 75\%. ${ }^{4-7}$ Approximately $70 \%-75 \%$ of CRC with LM are not suitable for hepatic resection with relatively poor short-term prognosis; chemotherapy plus biological therapies has been able to bring OS to $\sim 2$ years. ${ }^{8}$

Although the liver is the sole site of metastatic disease in as many as $40 \%$ of patients, LM is resectable in fewer than $20 \%{ }^{9}$ When surgery is not feasible, chemotherapy, radiofrequency ablation, intra-arterial chemotherapy and TACE are possible alternatives to achieve control of the disease in the liver. ${ }^{10}$ Irinotecan is a derivative of camptothecin, which kills cancer cells by inhibiting the function of the enzyme topoisomerase I, essential to DNA replication and employed in combined chemotherapy for first- and second-line treatment in patients with advanced colorectal cancer. Irinotecan can be loaded on drug-eluting beads to perform localized treatments because such beads allow a suitable release of the drug over time, prolonging the effect on tumor cells. In addition, drug-eluting beads can embolize the tumoral vascular bed leading to an ischemic effect. ${ }^{10,11}$

Recently, the use of DEBIRI has been reported with interesting results for the treatment of patients with LM from CRC previously treated with 2 or 3 lines of systemic therapy. ${ }^{11-13}$

The main aim of this prospective pilot study was to evaluate the tumor response of DEBIRI treatment for LM from $\mathrm{CRC}$ in patients who have failed second- and/or third-line systemic chemotherapy. Secondary aims were to evaluate the OS and the toxicity.

\section{Methods}

\section{Patients}

Twenty-five patients referred to the "Diagnostic and Interventional Radiology Unit with Integrated Section of Translational Medical Oncology" of the "National Cancer Research Centre" in Bari (Italy) from December 2011 to August 2014 were included in the study. Being a pilot study, the sample size was determined by a nonstatistical method. Patients of any race or sex were included for DEBIRI treatment if they were 18-80 years of age, with histologically confirmed CRC and unresectable LM occupying $<50 \%$ of the liver parenchyma calculated by the appropriate software on CT images, previously treated with 2 or 3 lines of chemotherapy, no radiological evidence of extrahepatic disease, adequate liver function (total bilirubin level $\leq 2.0 \mathrm{mg} / \mathrm{dL}, \mathrm{ALT}$ and AST $<5$ times upper limit of normal, albumin $\geq 2.5 \mathrm{~g} / \mathrm{dL}$ ), adequate hematologic function (absolute neutrophil count $\geq 1.5 \times 10^{9} / \mathrm{L}$, platelets $\geq 75 \times 10^{9} / \mathrm{L}$ and international normalized ratio $\leq 1.3$ without anticoagulants) and adequate renal function (serum creatinine $\leq 2.0 \mathrm{mg} / \mathrm{dL}$ ). All patients were fully staged using MDCT of the chest, abdomen and pelvis.

Patients were excluded in case of pregnancy, presence of severe cardiac comorbidities, history of HIV infection or if they had a previous or concurrent malignancy or a contraindication to angiographic and selective visceral catheterization.

A clinical and instrumental follow-up was performed 1 month after the DEBIRI treatment and then at 3-month intervals by clinical examinations, blood tests and thorax, abdomen and pelvis MDCTs.

Full ethical approval (code: 2011.61; date: December 13, 2011) from the ethical committee of the National Cancer Research Center of Bari and signed consent from individual patients were obtained to conduct the study.

\section{Interventional procedure and drug schedule}

DEBIRI consisted of drug-eluting beads (HepaSphere; BioSphere Medical, Roissy-en-France, France) loaded with irinotecan (Campto ${ }^{\circledR}$; Pfizer Italia, Milan, Italy) given at a dose of 100 or $200 \mathrm{mg} ;{ }^{14}$ a dose of $200 \mathrm{mg}$ was planned in case of bilobar involvement or if total target lesion size (sum of maximum diameters) was over $100 \mathrm{~mm}$.

Patients underwent DEBIRI administration using angiography. A catheter (Progreat 2.7 fr; Terumo Medical, Leuven, Belgium) was placed as selectively as possible in order to isolate the blood supply to the metastases and achieve localized chemotherapy.

Highly selective administration involved embolization of branches leading from the hepatic arteries, preferably the lesion itself or its feeding branches. The size of the drugeluting beads was chosen to be 100-300 $\mu \mathrm{m}$.

In case of whole liver involvement, the 2 lobes were treated during the same procedure.

Patients undergoing DEBIRI were monitored closely after each procedure in case hepatic side effects developed. Intravenous hydration, morphine, antiemetic and antibiotic prophylaxis were provided to reduce PES, which includes fever, abdominal pain and hypertransaminasemia.

Procedures were performed in general anesthesia according to previous literature reports, and morphine was administered in the postprocedure period. ${ }^{15-17}$ 
The number of treatments for each patient was decided after treatment response evaluation by MDCT at 1 month; in particular, only patients with PR or SD were retreated. The interval between treatments ranged from 1 to 2 months.

\section{Treatment response}

Treatment response assessment was performed by chest, abdomen and pelvis MDCT scan at 1 month from treatment completion, with evaluation of the enhancement pattern of the target lesion and tumor response rates according to mRECIST (Version 1.1) and previous literature regarding DEBIRI response clinical trials. ${ }^{10,18-20}$

Treatment response was categorized as CR, PR, SD and PD. CR was defined by the disappearance of measurable disease or the absence of arterial phase contrast enhancement as measured by MDCT, persisting for $\geq 4$ weeks without the appearance of new measurable lesions. PR was defined as $\mathrm{a} \geq 30 \%$ reduction in the sum of the products of the greatest diameter (length) and the greatest perpendicular diameter (width) of all measurable lesions compared with baseline, no appearance of new measurable lesions or reduction of arterial phase contrast enhancement. SD represented cases in which no differences from the baseline evaluation were observed. PD was defined by the occurrence of one of the following conditions: the sum of the cross products of all measurable lesions, including new lesions, increases by $>50 \%$ compared with the nadir or new measurable lesions occur in any part of the body outside the liver.

\section{Toxicity evaluation}

The toxicity profile was assessed based on patient's symptoms following each procedure. Toxic effects experienced up to 7 days after each treatment were considered in the toxicity evaluation and were monitored until disappearance. In cases of G3 and G4 hypertransaminasemia, patients were monitored until transaminase levels became lower than $2 x$ upper normal values.

All adverse events were recorded per standards and terminology set forth by the Cancer Therapy Evaluation Program Common Terminology Criteria for Adverse Events, Version 3.0.

\section{Statistical analysis}

Associations of tumor response and variables were calculated by the chi-squared test. OS was calculated using the Kaplan-Meier method from the date of the first liver DEBIRI, performed within 1 week of enrollment, to the date of last follow-up or death. Comparisons were made using the log-rank test. To identify factors associated with OS in the entire cohort, variables were assessed using the Cox regression model. The $P$-value $<0.05$ was considered significant. All statistical analyses were performed using SPSS Statistics software (Version 2.0).

\section{Results \\ Patients and treatments}

Patients' clinicopathological characteristics are reported in Table 1.

A total of 25 patients underwent from 1 to 3 DEBIRI treatments for a total of 44 treatment sessions. All patients received at least 2 lines of chemotherapy before DEBIRI (FOLFOX and/or FOLFIRI plus biological therapy according to Kras status).

All patients underwent primary colon cancer resection. Sixteen patients had synchronous LM (6/16 patients in the right liver and 10/16 in the whole liver) and 5 of them also underwent hepatic surgery; in 9/25 patients metachronous

Table I Clinicopathological characteristics of patients

\begin{tabular}{ll}
\hline Variables & $\mathbf{n}(\%)$ \\
\hline Sex & \\
Male & $16 / 25(64)$ \\
Female & $9 / 25(36)$ \\
Age (years) & \\
Range & $43-80$ \\
Mean & 62.72 \\
Median & 59 \\
Primary adenocarcinoma sites & \\
Right colon & $3 / 25(12)$ \\
Left colon & $7 / 25(28)$ \\
Sigma & $9 / 25(36)$ \\
Rectum & $6 / 25(24)$ \\
Dukes' Classification (Astler-Coller modification) & \\
A & $2(8)$ \\
B & $2(8)$ \\
C & $6(24)$ \\
D & $15(60)$ \\
Grading & \\
GI & $6 / 25(24)$ \\
G2 & $12 / 25(48)$ \\
G3 & $7 / 25(28)$ \\
Kras & \\
Wild type & $17 / 25(68)$ \\
Mutated & $8 / 25(32)$ \\
2 & \\
3 & $19 / 25(76)$ \\
Number of DEBIRI treatments & $6 / 25(24)$ \\
I & \\
2 & $10 / 25(40)$ \\
3 & $11 / 25(44)$ \\
\hline Abbremotherapy before DEBIRI & $4 / 25(16)$ \\
\hline
\end{tabular}

Abbreviation: DEBIRI, drug-eluting beads with irinotecan. 
LM appeared (2/9 in the right liver, 2/9 in the left liver and $5 / 9$ in the whole liver) and 2 of them (1 with bilobar LM and 1 with left lobe LM) underwent hepatic lobectomy. Patients were hospitalized for a median time of 10 days (range: 7-28). DEBIRI treatments and hepatic lesion characteristics are reported in Table 2. A total of $>113$ lesions were treated; in particular 1 hepatic lesion was involved in 12/37 treatments, 2 in 1/37 treatments, 3 in 7/37 treatments, 4 in 7/37 treatments and multiple $(\geq 5)$ in $10 / 37$ treatments.

\section{Treatment response}

This analysis was performed considering 37/44 treatments in 23/25 patients, as for 2/44 treatments (in 2/25 patients), MDCT performed within 1 month of DEBIRI treatment was not available, with the CT scan being performed with an older generation device, because of the maintenance of the routinely used device; for 5/44 treatments, the timing of MDCT was not respected, so the images were not suitable for the comparison with the pretreatment images and were not included in this analysis.

According to mRECIST, considering the 23 patients, CR was observed in $5(21.8 \%)$ patients, $\mathrm{PR}$ in $3(13 \%), \mathrm{SD}$ in $12(52.2 \%)$ and $\mathrm{PD}$ in $3(13 \%)$ patients. The response rate $(\mathrm{RR}=\mathrm{CR}+\mathrm{PR})$ was $34.8 \%$. MDCT images of a complete responder patient are reported in Figure 1. Angiography images of pre- and postembolization are reported in Figure 2.

Considering the 37 DEBIRI treatments, CR was observed in $6 / 37(16.2 \%)$ treatments, PR in $6 / 37(16.2 \%)$, SD in $22 / 37(59.5 \%)$ and PD in $3 / 37$ (8.1\%) treatments. Patients underwent more than 1 DEBIRI when they showed PR or SD. No associations between treatment response and variables such as Dukes' classification, grading, Kras status, lesion size, number of lesions, number of DEBIRI

Table 2 DEBIRI treatments and hepatic lesion characteristics

\begin{tabular}{ll}
\hline Variables & $\mathbf{n ~ ( \% )}$ \\
\hline $\begin{array}{l}\text { Doses of DEBIRI (median I00 mg) } \\
\text { I00 mg }\end{array}$ & $26 / 44$ (59) treatments \\
$200 \mathrm{mg}$ & $18 / 44(4 \mathrm{I})$ treatments \\
$\begin{array}{l}\text { Sites of treatments } \\
\text { Right liver }\end{array}$ & $36 / 44$ (8I.8) treatments \\
Left liver & $6 / 44$ (I3.6) treatments \\
Whole liver & $2 / 44(4.6)$ treatments \\
Number of hepatic & Median: 3; range: I-20 \\
lesions for treatment & \\
Hepatic lesions size & Mean: 3I.86 mm; range: 8-II5 mm \\
Total target lesion size (sum & 88 mm; range: I0-240 mm \\
of a maximum of 5 lesions) & \\
\hline
\end{tabular}

Abbreviation: DEBIRI, drug-eluting beads with irinotecan. treatments for each patients and doses of DEBIRI were found $(P>0.05)$.

\section{OS}

Follow-up range was 4-42 months. At the end of the follow-up, 15/25 (60\%) patients were alive while 10/25 had died (40\%). The median OS was 37 months (95\% CI: 13.881 to 60.119 ; Figure 3 ).

The Cox regression model showed that neither size, number of lesions, Dukes' classification, grading, Kras status nor number of chemotherapy pre-DEBIRI influenced the $\mathrm{OS}$, even if these results could be influenced by the small number of patients.

The log-rank test showed no statistically significant difference in OS among patients who underwent 1, 2 or 3 DEBIRI treatments $\left(\chi^{2}=2.831, P=0.09\right)$. In 6 patients, extrahepatic metastases occurred.

\section{Toxicity}

Toxicity results concerning all the 44 DEBIRI treatments are reported in Table 3.

No associations between dose and fever, AST and ALT, respectively, and number of DEBIRI were found $(P>0.05)$. No associations between tumor response and fever, AST and ALT, respectively, were found $(P>0.05)$.

Furthermore, 1 abscess was observed after 1 DEBIRI and was considered a procedure-related major complication.

\section{Discussion}

Systemic chemotherapy for unresectable LM from CRC is the standard initial management. First-line and second-line chemotherapies consist of 5-fluorouracil and leucovorin combining irinotecan or oxaliplatin. Patients with Kras wildtype status can benefit from a combination of chemotherapy agent plus cetuximab; in these patients, a third-line therapy is possible following a replacement among EGFR inhibitors. In patients with a Kras mutation, first- and second-line chemotherapies are accepted with the addition of bevacizumab. Currently, the median OS for systemic chemotherapies associated with biological agents is around 24 months; it should be considered, however, that each subsequent line of systemic chemotherapy is associated with a worsening of adverse events. ${ }^{5,20-22}$

In patients with confined LM who failed systemic lines of therapy, locoregional therapy employing TACE is an important treatment option. Classical TACE involves the emulsification of a chemotherapeutic agent in an oily medium, used 

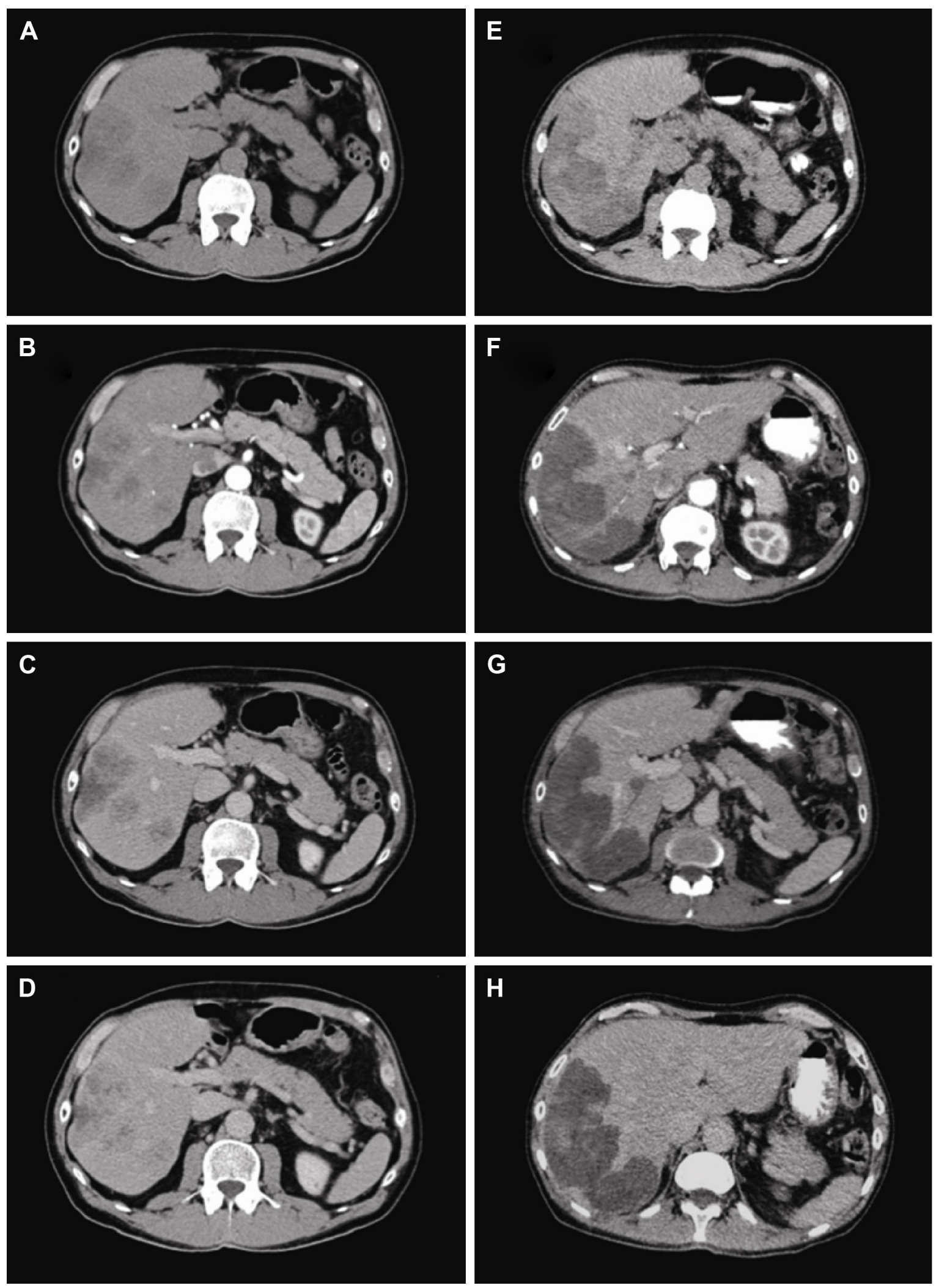

Figure I MDCT baseline and post-DEBIRI treatment in a complete responder patient.

Notes: A 74-year old patient with right colon cancer, mutated Kras status, who performed 2 lines of systemic chemotherapy. Baseline MDCT axial images (A-D) showed multiple hypodense liver metastases. MDCT performed I month after DEBIRI treatment $(\mathbf{E}-\mathbf{H})$ showed the same hypodense lesions without contrast enhancement. The patient was considered as a complete responder. OS was 24 months and the patient showed GI fever and GI hypertransaminasemia.

Abbreviations: MDCT, multidetector contrast enhancement computed tomography; DEBIRI, drug-eluting beads with irinotecan; OS, overall survival. 

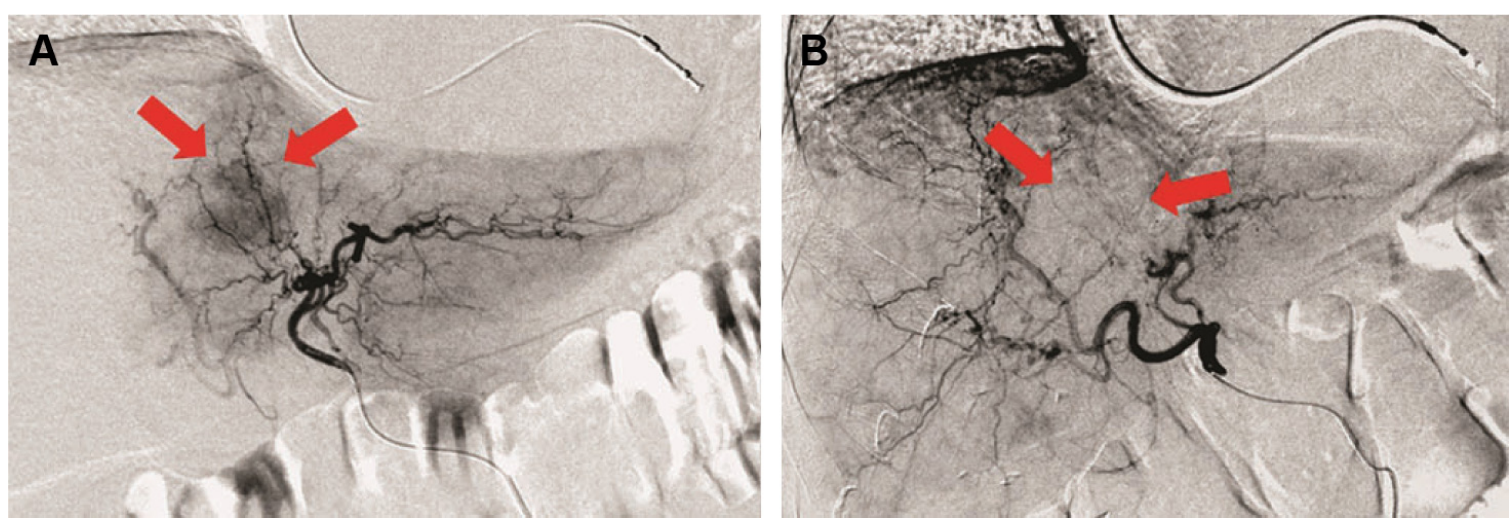

Figure 2 Angiography pre- and post-DEBIRI treatment in a complete responder patient.

Notes: A 60-year old patient with single metastasis from colorectal cancer in the III-IV segment of the left lobe of the liver. In the pretreatment angiography (A) the red arrows show the moderate and diffuse vascularization of the unencapsulated lesion with irregular margins. In the posttreatment angiography (B) the red arrows show the lack of the vascularization in the site of the lesion as a complete response to the treatment. In the upper part of the figure (A and $\mathbf{B}$ ), the dual chamber cardiac catheter of the pacemaker is evident.

Abbreviation: DEBIRI, drug-eluting beads with irinotecan.

in this case as a drug carrier, which is delivered intra-arterially to the liver tumor. ${ }^{14}$

TACE produces a shutdown of blood flow and the simultaneous release of high doses of the drug, increasing the drug concentration and exposure. . $^{23,24}$

TACE with DEBIRI was first reported for the treatment of LM in 2006. ${ }^{11}$ The primary function of the device is to embolize the arteries feeding the tumor site, causing nutrient and oxygen starvation of the tumor and thereby inducing necrosis in the tumor tissue. The secondary function is to deliver irinotecan in a controlled manner to tumor sites. ${ }^{5}$

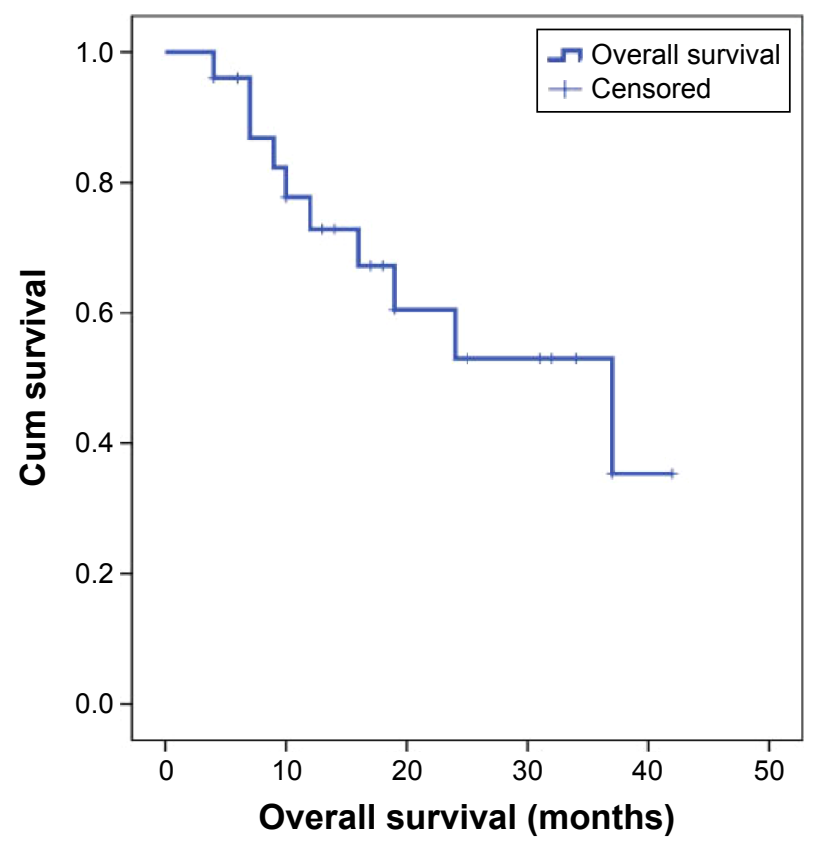

Figure 3 OS curve for 25 patients who underwent DEBIRI treatment.

Abbreviations: OS, overall survival; DEBIRI, drug-eluting beads with irinotecan; Cum, cumulative.
A consequence of hypoxia caused by embolization is the adaptive response of increasing production of VEGF that stimulates angiogenesis; this post-TACE-induced pathway could determine the regrowth of the tumor. ${ }^{14,25}$

Although DEBIRI therapy is not currently approved by the most important international guidelines, there is an increasing body of data from clinical trials. ${ }^{26}$

In line with previously published analyses, we evaluated the response to therapy at 1 month after DEBIRI treatment. ${ }^{10,20,27}$

Literature reports RR varying from $36 \%$ to $78 \%{ }^{26}$ Fiorentini et $\mathrm{al}^{10}$ showed that in 35 patients RR was $68.6 \%$, SD was $11.4 \%$ and PD was $20 \%$. Narayan et $\mathrm{al}^{20}$ showed that in 20 patients, $15 \%$ of patients were CR, $30 \%$ were PR, $20 \%$ were $\mathrm{SD}$ and $35 \%$ were PD. Jones et $\mathrm{al}^{27}$ showed that in 22 patients, $91 \%$ were $\mathrm{SD}$ and $9 \%$ were $\mathrm{PD}$.

The results of our study are in line with those reported in the literature, with a slightly lower RR (34.8\%) and similar SD and PD (52.2 and 13\%). The wide spectrum of literature results could be explained by the small number of patients in each study and the heterogeneity in the different studies regarding the number of DEBIRI treatments, the dose administered and the number and the extension of LM. To the best of our knowledge, no previous studies have reported a relationship between tumor response and number of DEBIRI treatments, dose or number and extension of LM; in our study, these variables did not influence the tumor response.

Improvement of OS is one of the goals achievable with DEBIRI treatment. In the only small Phase III study published by Fiorentini et al, ${ }^{10}$ the survival advantage conferred by DEBIRI over systemic chemotherapy (22 vs 15 months) suggests that DEBIRI may represent an interesting clinical treatment modality. Our preliminary data indicate an 
Table 3 Toxicity results concerning the 44 DEBIRI treatments

\begin{tabular}{|c|c|c|c|c|c|c|c|c|}
\hline \multirow{2}{*}{$\begin{array}{l}\text { Toxicity (\% out } \\
\text { of } 44 \text { treatments) }\end{array}$} & \multicolumn{2}{|l|}{ GI } & \multicolumn{2}{|l|}{ G2 } & \multicolumn{2}{|l|}{ G3 } & \multicolumn{2}{|l|}{ G4 } \\
\hline & $100 \mathrm{mg}$ & $200 \mathrm{mg}$ & $100 \mathrm{mg}$ & $200 \mathrm{mg}$ & $100 \mathrm{mg}$ & $200 \mathrm{mg}$ & $100 \mathrm{mg}$ & $200 \mathrm{mg}$ \\
\hline Pain & $6 \%$ & $7 \%$ & $3 \%$ & $4 \%$ & $1 \%$ & $1 \%$ & $0 \%$ & $0 \%$ \\
\hline Hypertension & $9 \%$ & $10 \%$ & $5 \%$ & $6 \%$ & $0 \%$ & $0 \%$ & $0 \%$ & $0 \%$ \\
\hline Vomiting & $2 \%$ & $3 \%$ & $0 \%$ & $2 \%$ & $0 \%$ & $0 \%$ & $0 \%$ & $0 \%$ \\
\hline Nausea & $0 \%$ & $0 \%$ & $0 \%$ & $0 \%$ & $0 \%$ & $0 \%$ & $0 \%$ & $0 \%$ \\
\hline Diarrhea & $0 \%$ & $2 \%$ & $0 \%$ & $0 \%$ & $0 \%$ & $0 \%$ & $0 \%$ & $0 \%$ \\
\hline Asthenia & $0 \%$ & $0 \%$ & $0 \%$ & $0 \%$ & $0 \%$ & $0 \%$ & $0 \%$ & $0 \%$ \\
\hline Leukopenia & $3 \%$ & $4 \%$ & $3 \%$ & $4 \%$ & $0 \%$ & $0 \%$ & $0 \%$ & $0 \%$ \\
\hline Anemia & $3 \%$ & $3 \%$ & $2 \%$ & $3 \%$ & $0 \%$ & $0 \%$ & $0 \%$ & $0 \%$ \\
\hline Fever & $8 \%$ & $8 \%$ & $0 \%$ & $0 \%$ & $0 \%$ & $0 \%$ & $0 \%$ & $0 \%$ \\
\hline Alopecia & $0 \%$ & $0 \%$ & $0 \%$ & $0 \%$ & $0 \%$ & $0 \%$ & $0 \%$ & $0 \%$ \\
\hline Hypertransaminasemia (AST) & $11 \%$ & $12 \%$ & $11 \%$ & $12 \%$ & $11 \%$ & $12 \%$ & $0 \%$ & $2 \%$ \\
\hline Hypertransaminasemia (ALT) & $15 \%$ & $15 \%$ & $8 \%$ & $10 \%$ & $8 \%$ & $8 \%$ & $0 \%$ & $2 \%$ \\
\hline
\end{tabular}

Abbreviations: DEBIRI, drug-eluting beads with irinotecan; AST, aspartate aminotransferase; ALT, alanine aminotransferase.

interesting median OS of 37 months, but this result has to be considered with caution due to the pilot nature of our study, the small number of patients and the absence of randomization.

An important potential advantage of DEBIRI treatment is the minimal onset of systemic side effects thanks to the localized delivery of the drug. The good tolerability of DEBIRI is an important goal, especially in heavily pretreated patients. The most common adverse event is PES, which involves abdominal pain, nausea and vomiting. This was reported as mild in the studies of Eichler et $\mathrm{al}^{28}$ and Martin et $\mathrm{al}^{29}$ and made up $40 \%-63 \%$ of adverse events. ${ }^{5}$ PES was the most common adverse event also in our study (Table 3 ); in cases of G3 pain, we administered morphine after the procedure.

Hypertension is the second most common adverse event reported and it seems related to pain (the most common complication), as it was transitory and self-limiting with a temporal coincidence during the postprocedural course. Eichler et $\mathrm{al}^{28}$ reported hypertension as making up $<10 \%$ of adverse events, and Martin et $\mathrm{al}^{29}$ reported it as making up $4 \%$ of adverse events. Also in our study, mild transitory hypertension was observed after $30 \%$ of treatments, and it was likely related to pain and postanesthesia hydration and in some cases to corticosteroid administration.

There were signs of increased liver enzymes in most patients, but these were transient; in our analysis, hypertransaminasemia was not related to fever. ${ }^{5}$ Adverse events in the more serious category were rare. In a review of 2300 TACE procedures for various indications, Sakamoto et $\mathrm{al}^{30}$ reported 102 complications (4.4\%), of which 39 were related to catheter dissections or perforations. In our study, the only most serious adverse event was the abscessualization of the treated lesion that occurred only after 1 TACE and was treated with drainage. This was the case of the longer hospitalization (28 days). Our median time of hospitalization was 10 days, longer than those reported in the literature ${ }^{10}$ due to the treatment being performed in general anesthesia, differently to the cited studies. We preferred general anesthesia to guarantee the complete immobilization of the patients during the DEBIRI procedure.

Furthermore, the longer hospitalization time in our experience was also due to the cases in which hypertransaminasemia (G3 and G4) appeared because we prefer to discharge patients only when transaminase levels are lower than $2 \times$ normal values.

Adequate supportive treatment with antibiotic and antiemetic prophylaxis and intravenous hydration was strictly necessary until the serum levels of transaminases were stabilized in order to prevent infections.

Only Martin et $\mathrm{al}^{31}$ in their multivariate analysis in 109 patients identified the $\geq 3$ DEBIRI treatments and dose $>100 \mathrm{mg}$ as risk factors for toxicity. In our study, none of them influenced the onset of adverse events, even if we should remember that only $4 / 25$ patients performed 3 DEBIRI treatments, while the dose administered was $200 \mathrm{mg}$ in $41 \%$ of treatments. In our study, no patients performed more than 3 DEBIRI treatments due to the onset of extrahepatic metastasis, serious adverse events or worsening of performance status. To the best of our knowledge, no previous studies have investigated the role of toxic effects as possible predictors of tumor response; in the analysis we performed neither fever nor hypertransaminasemia was related to it.

\section{Conclusion}

DEBIRI is an effective and a well-tolerated therapy in patients with LM from CRC who have failed second- and/or third-line systemic chemotherapy plus biological agents. The favorable tumor response and the favorable toxicity profile make this local treatment a potential third-line therapy. 
Our results should be interpreted with caution due to the small number of enrolled patients related to the pilot nature of the study. Further larger studies are needed to confirm these preliminary data to consider DEBIRI as an emerging attractive treatment in these patients.

Furthermore, to improve DEBIRI results, the combination with angiogenic inhibitors should be investigated to prevent angiogenic rebound.

\section{Acknowledgments}

The authors greatly appreciate the optimal support from Dr Francesco Armenise, Dr Mariangela Porcelli, Dr Giuseppe Laricchia and Dr Pasquale Molinari, and the language editing by Caroline Oakley.

\section{Author contributions}

GR conceived the study and was its principal coordinator; ANA participated in the study coordination and contributed to the revision of the manuscript; CA participated in study conception, developing and writing of the manuscript; LC, IM and GV selected cases, participated in data interpretation and in patient clinical follow-up; MF performed statistical analysis. VF, FM and DdC performed DEBIRI treatment and reviewed radiological records; MA participated at the performance of DEBIRI treatments; GR gave his important intellectual content and assisted in writing the manuscript; CDG performed and coordinated the DEBIRI treatments, gave his fundamental intellectual content and the final approval of the version to be published. All authors contributed toward data analysis, drafting and critically revising the paper and agree to be accountable for all aspects of the work.

\section{Abbreviations}

CRC, colorectal cancer; LM, liver metastases; OS, overall survival; TACE, transarterial chemoembolization; DEBIRI, drug-eluting beads with irinotecan; MDCT, multidetector contrast enhancement computed tomography; mRECIST, modified Response Evaluation Criteria in Solid Tumor; CR, complete response; $\mathrm{PR}$, partial response; $\mathrm{SD}$, stable disease; $\mathrm{PD}$, progressive disease; RR, response rate; PES, postembolization syndrome; ALT, alanine aminotransferase; AST, aspartate aminotransferase; CT, computed tomography.

\section{Disclosure}

The authors report no conflicts of interest in this work.

\section{References}

1. Parkin DM, Bray F, Ferlay J, Pisani P. Global cancer statistics, 2002. CA Cancer J Clin. 2005;55(2):74-108.
2. Welch JP, Donaldson GA. The clinical correlation of an autopsy study of recurrent colorectal cancer. Ann Surg. 1979;189(4):496-502.

3. Scheele J, Stangl R, Altendorf-Hofmann A, Gall FP. Indicators of prognosis after hepatic resection for colorectal secondaries. Surgery. 1991; 110(1):13-29.

4. Kemeny NE, Kemeny MM, Lawrence TS. Liver Metastases. 3th ed. Philadelphia, PA: Elsevier Clinical Oncology; 2004:1141-1178.

5. Martin RCG, Joshi J, Robbins K, et al. Hepatic intra-arterial injection of drug-eluting bead, irinotecan (DEBIRI) in unresectable colorectal liver metastases refractory to systemic chemotherapy: results of multiinstitutional study. Ann Surg Oncol. 2011;18(1):192-198.

6. Fong Y, Fortner J, Sun RL, Brennan MF, Blumgart LH. Clinical score for predicting recurrence after hepatic resection for metastatic colorectal cancer: analysis of 1001 consecutive cases. Ann Surg. 1999;230(3): 309-318. [discussion 318-321].

7. Bhattacharjya S, Aggarwal R, Davidson BR. Intensive follow-up after liver resection for colorectal liver metastases: results of combined serial tumour marker estimations and computed tomography of the chest and abdomen - a prospective study. Br J Cancer. 2006;95(1):21-26.

8. Saltz LB, Lenz HJ, Kindler HL, et al. Randomized phase II trial of cetuximab, bevacizumab, and irinotecan compared with cetuximab and bevacizumab alone in irinotecan-refractory colorectal cancer: the BOND-2 study. J Clin Oncol. 2007;25(29):4557-4561.

9. Manfredi S, Lepage C, Hatem C, Coatmeur O, Faivre J, Bouvier AM. Epidemiology and management of liver metastases from colorectal cancer. Ann Surg. 2006;244(2):254-259.

10. Fiorentini G, Aliberti C, Tilli M, et al. Intra-arterial infusion of irinotecan-loaded drug-eluting beads (DEBIRI) versus intravenous therapy (FOLFIRI) for hepatic metastases from colorectal cancer: final results of a phase III study. Anticancer Res. 2012;32(4):1387-1396.

11. Aliberti C, Tilli M, Benea G, Fiorentini G. Trans-arterial chemoembolization (TACE) of liver metastases from colorectal cancer using irinotecan-eluting beads: preliminary results. Anticancer Res. 2006; 26(5B):3793-3796.

12. Fiorentini G, Aliberti C, Turrisi G, et al. Intra-arterial hepatic chemoembolization of liver metastases from colorectal cancer adopting irinotecan-eluting beads: results of a phase II clinical study. In Vivo. 2007;21(6):1085-1091.

13. Fiorentini G, Aliberti C, Montagnani F, Tilli M, Mambrini A, Benea G. Trans-arterial chemoembolization of metastatic coloretal carcinoma (MCRC) to the liver adopting polyvinyl alcohol microspheres (PAM) loaded with irinotecan compared with FOLFIRI (CT): evaluation at two years of a phase III clinical trial. Ann Oncol. 2010;21(suppl 89):198-224.

14. Gadaleta CD, Ranieri G. Trans-arterial chemoembolization as a therapy for liver tumours: new clinical developments and suggestions for combination with angiogenesis inhibitors. Crit Rev Oncol Hematol. 2011; 80(1):40-53.

15. Daniele A, Divella R, Quaranta M, et al. Clinical and prognostic role of circulating MMP-2 and its inhibitor TIMP-2 in HCC patients prior to and after trans-hepatic arterial chemo-embolization. Clin Biochem. 2014;47(3):184-190.

16. Gadaleta CD, Solbiati L, Mattioli V, et al. Unresectable lung malignancy: combination therapy with segmental pulmonary arterial chemoembolization with drug-eluting microspheres and radiofrequency ablation in 17 patients. Radiology. 2013;267(2):627-637.

17. Gadaleta CD, Catino A, Ranieri G, Armenise F, Console G, Mattioli V. Hypoxic stop-flow perfusion with mitomycin-C in the treatment of multifocal liver metastases. Usefulness of a vascular arterial stent to prevent iatrogenic lesions of the hepatic arterial wall. J Exp Clin Cancer Res. 2003;22(suppl 4):203-206.

18. Eisenhauer EA, Therasse P, Bogaerts J, et al. New response evaluation criteria in solid tumours: revised RECIST guideline (version 1.1). Eur J Cancer. 2009;45(2):228-247.

19. Lencioni R, Llovet J. Modified RECIST (mRECIST) assessment for hepatocellular carcinoma. Semin Liver Dis. 2010;30(1):52-60.

20. Narayan G, Barbery K, Suthar R, Guerrero G, Arora G. Transarterial chemoembolization using DEBIRI for treatment of hepatic metastases from colorectal cancer. Anticancer Res. 2013;33(5):2077-2084. 
21. Karapetis C, Khambata-Ford S, Jonker D, et al. K-RAS mutations and benefit from cetuximab in advanced colorectal cancer. $N$ Engl J Med 2008;359(17):1757-1765.

22. Chen HX, Mooney M, Boron M, et al. Phase II multicenter trial of bevacizumab plus fluorouracil and leucovorin in patients with advanced refractory colorectal cancer: an NCI Treatment Referral Center Trial TRC-0301. J Clin Oncol. 2006;24(21):3354-3360.

23. Fiorentini G. A new tool to enhance the efficacy of chemoembolization to treat primary and metastatatic hepatic tumors. Expert Opin Drug Deliv. 2011;8(4):409-413.

24. Vogl TJ, Zangos S, Eichler K, Yakoub D, Nabil M. Colorectal liver metastases:regional chemotherapy via transarterial chemoembolization (TACE) and hepatic chemoperfusion: an update. Eur Radiol. 2007; 17(4):1025-1034.

25. Ranieri G, Patruno R, Ruggieri E, Montemurro S, Valerio P, Ribatti D. Vascular endothelial growth factor (VEGF) as a target of bevacizumab in cancer: from the biology to the clinic. Curr Med Chem. 2006; 13(16):1845-1857.

26. Richardson AJ, Laurence JM, Lam VW. Transarterial chemoembolization with irinotecan beads in the treatment of colorectal liver metastases: systematic review. J Vasc Interv Radiol. 2013;24(8):1209-1217.
27. Jones RP, Stattner S, Dunne DFJ, et al. Radiological assessment of response to neoadjuvant transcatheter hepatic therapy with irinotecaneluting beads (DEBIRI ${ }^{\circledR}$ ) for colorectal liver metastases does not predict tumour destruction or long-term outcome. EJSO. 2013;39(10): 1122-1128.

28. Eichler K, Zangos S, Mack MG, et al. First human study in treatment of unresectable liver metastases from colorectal cancer with irinotecanloaded beads (DEBIRI). Int J Oncol. 2012;41(4):1213-1220.

29. Martin RC, Scoggins CR, Tomalty D, et al. Irinotecan drug-eluting beads in the treatment of chemo-naïve unresectable colorectal liver metastasis with concomitant systemic fluorouracil and oxaliplatin: results of pharmacokinetics and phase I trial. J Gastrointest Surg. 2012;16(8): 1531-1538.

30. Sakamoto I, Aso N, Nagaoki K, et al. Complications associated with transcatheter arterial embolization for hepatic tumors. Radiographics. 1998;18(3):605-619.

31. Martin RC, Howard J, Tomalty D, et al. Toxicity of irinotecaneluting beads in the treatment of hepatic malignancies: results of a multi-institutional registry. Cardiovasc Intervent Radiol. 2010;33(5): 960-966.
OncoTargets and Therapy

\section{Publish your work in this journal}

OncoTargets and Therapy is an international, peer-reviewed, open access journal focusing on the pathological basis of all cancers, potential targets for therapy and treatment protocols employed to improve the management of cancer patients. The journal also focuses on the impact of management programs and new therapeutic agents and protocols on

\section{Dovepress}

patient perspectives such as quality of life, adherence and satisfaction. The manuscript management system is completely online and includes a very quick and fair peer-review system, which is all easy to use. Visit http://www.dovepress.com/testimonials.php to read real quotes from published authors. 